\title{
Electron transport through a mesoscopic hybrid multiterminal resonant-tunneling system
}

\author{
Qing-feng Sun \\ Department of Physics, The University of Hong Kong, Pokfulam Road, Hong Kong, China \\ Department of Physics, Peking University, Beijing 100871, China \\ Bai-geng Wang and Jian Wang \\ Department of Physics, The University of Hong Kong, Pokfulam Road, Hong Kong, China \\ Tsung-han Lin* \\ Department of Physics, The University of Hong Kong, Pokfulam Road, Hong Kong, China \\ and Department of Physics, Peking University, Beijing 100871, China
}

(Received 2 August 1999)

\begin{abstract}
For a mesoscopic hybrid system which contains a normal central region coupled to multiple superconducting leads, a general expression of the current is derived by using nonequilibrium-Green-function method. This current formula can be used to describe the case with time-dependent external fields applied to any parts of the system, arbitrarily finite voltages, and any kinds of interactions in the central region. For a normal two-terminal interacting electron system, this expression reduces to the general time-dependent current formula previously obtained by Wingreen et al. [Phys. Rev. B 98, 8487 (1993)]. As an application, we use this current formula to study a special case of a noninteracting single-level central region coupled to two or three superconducting leads, respectively.
\end{abstract}

\section{INTRODUCTION}

Mesoscopic physics, a branch of condensed-matter physics, was developed and became an active field over the last two decades. The quantum transport property of mesoscopic systems is one of the most striking phenomena. Because of the possibility of designing and fabricating artificial structures, studies on transport are no longer limited to the systems provided by nature, and have opened an extremely rich field for basic and applied researches. ${ }^{1}$

Recently, electron tunneling through a mesoscopic system, which is applied by external time-dependent fields, has been attracting more and more attention. The essential feature of mesoscopic physics is the phase coherence of the charge carriers. Generally, the external time-dependent perturbation affects the phase factor of the wave function differently in different parts of the system, ${ }^{2,3}$ leading to wellknown photon-assisted tunneling (PAT), in which an electron can tunnel through the system by emitting or absorbing photons. Such a PAT process is responsible for the sideband peaks in the curve of the conductance vs the gate voltage and the substep structure in the current-bias-voltage $(I-V)$ characteristics. PAT has been investigated experimentally and theoretically in a variety of systems, including a single quantum dot, ${ }^{4,5}$ two coupled quantum dots, ${ }^{6}$ semiconductor superlattices, ${ }^{7}$ etc. In particular, Wingreen et al. presented a general formalism for electron transport through an interacting resonant-tunneling system in the presence of time-dependent external fields by using the nonequilibrium-Green-function method under the adiabatic approximation. $^{2,3}$

If the mesoscopic system contains superconducting components, usually called the mesoscopic "hybrid" systems, the situation will be more interesting. Basic features origi- nated from both mesoscopics and superconductivity participate jointly in the physics, which makes these "hybrid" systems a very fruitful research field. ${ }^{8}$ Many interesting phenomena have been studied for various mesoscopic "hybrid" systems: the subharmonic gap struture in $S-I-S$ or $S-N$ $S$ junctions, where $S, I$, and $N$ indicate superconductor, insulator, and normal metal, respectively, ${ }^{9}$ the Andreev bound states in $S-N-S$ or $N-I-N-S$ systems, ${ }^{10}$ the even-odd-parity asymmetry and the Coulomb blockade of the Andreev reflection in superconductor-superconducting-quantum-dotsuperconductor ( $S$-SQD- $S$ ) or $N$-SQD- $N$ systems, ${ }^{11}$ the dc or ac Josephson effect in $S-N-S$ or $S$-NQD- $S$ systems, ${ }^{12-16}$ etc.

In this paper, we investigate electron transport through a mesoscopic normal central region coupled to multiply superconducting or normal leads under the influence of timedependent external fields. We derive a formal expression for the fully nonlinear, time-dependent current through the system by the nonequilibrium-Green-function method. The formalism permits arbitrary interactions in the central region. For a two-terminal interacting system in a normal state, the current expression obtained reduces to the general timedependent current formula presented by Wingreen and co-workers. ${ }^{2,3}$ Then, as an example, we study the dc Josephson effect analytically and numerically, for the case in which the central region is a noninteracting single-level quantum dot and without the external fields. Two special cases with two or three superconducting terminals are investigated in detail. For the two-terminal system, the Josephson current is obtained, which consists of two parts: $I^{c}$ from the continuous spectrum, and $I^{d}$ from the discrete spectrum. For the threeterminal system, we investigate the relation between the dc Josephson current and the phases $\Phi_{i}^{0}(i=1,2,3)$ of the $i$ th superconducting lead. 


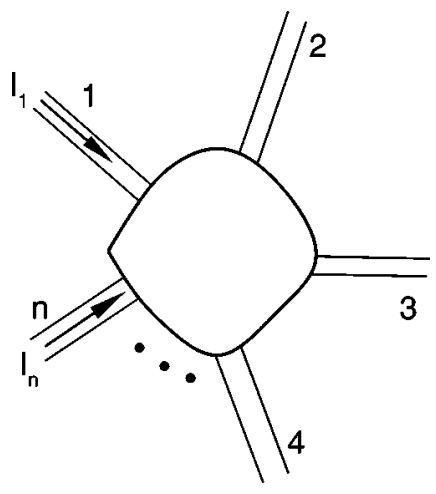

FIG. 1. A schematic diagram for the multiterminal mesoscopic hybrid system under consideration.

The outline of this paper is as follows. In Sec. II, the model Hamiltonian is presented. In Sec. III, we derive a general current formula for mesoscopic "hybrid" multiterminal resonant-tunneling systems, and discuss the relation between this current expression and the general timedependent current formula previously obtained by Wingreen et al. We also obtain the expression of the equilibrium current (i.e., the dc Josephson current) for the case of zero bias voltage and without external time-dependent fields. In Sec. IV, we use our general current formula to study the dc Josephson current through a noninteracting single-level central region. Two special cases, with either two or three terminals (superconducting leads), are studied. Finally, a brief summary is presented in Sec. V.

\section{MODEL}

The multiterminal resonant-tunneling system under consideration is shown in Fig. 1. The total Hamiltonian is split into three parts: $H(t)=H_{\text {leads }}(t)+H_{c e n}(t)+H_{T}(t)$, where $H_{\text {leads }}$ models multiterminal superconducting (or normal) electrodes, $H_{c e n}$ is for the central region, and $H_{T}$ describes the tunneling part between leads and the central region, respectively.

Here we assume that the $n$th lead is a BCS superconductor with the order parameter $\Delta_{n}$, which can also describe the normal lead of noninteracting electrons just by taking $\Delta_{n}=0$. The time-dependent external fields applied to the system are treated by the adiabatic approximation, ${ }^{2,3}$ i.e., the external fields only affect the quasiparticle energies $\epsilon_{n k}(t)$ (where the index $n$ means the $n$th lead, and $k$ is the index representing the quantum state) and the Cooper pair energies, but the occupation number of particles at each state $(n, k)$ remains unchanged. The single-particle energy $\epsilon_{n k}(t)$, which is time dependent under the external fields, can be separated into three parts: $\epsilon_{n k}(t)=\epsilon_{n k}^{0}+\mu_{n}(t)=\epsilon_{n k}^{0}+q V_{n}+W_{n}(t)$, where $\epsilon_{n k}^{0}$ is the time-independent single-particle energy without the time-dependent external fields and the dc bias voltage, $V_{n}$ is the dc bias voltage on the $n$th lead, $W_{n}(t)$ is a time-dependent part from the external field, and $q$ is the charge of the electron. Thus the Hamiltonian of all superconducting leads, $H_{\text {leads }}(t)$ is ${ }^{14,17}$

$$
\begin{aligned}
H_{l e a d s}(t)= & \sum_{n, k, \sigma} \epsilon_{n k}(t) a_{n k \sigma}^{\dagger} a_{n k \sigma}+\sum_{n, k}\left[\tilde{\Delta}_{n} a_{n k \downarrow} a_{n,-k \uparrow}\right. \\
& \left.+\widetilde{\Delta}_{n}^{*} a_{n,-k \uparrow}^{\dagger} a_{n k \downarrow}^{\dagger}\right],
\end{aligned}
$$

where $a_{n k \sigma}^{\dagger}\left(a_{n k \sigma}\right)$ are the creation (annihilation) operators of the electron in the $n$th lead. It should be emphasized that in the presence of the external fields, not only the energy of the quasiparticle, but also the energy of the Cooper pair, is affected, ${ }^{18}$ so the complex superconducting order parameter $\widetilde{\Delta}_{n}$ becomes

$$
\widetilde{\Delta}_{n}=\Delta_{n} \exp \left[-\frac{2 i}{\hbar} \int_{0}^{t} \mu_{n}(\tau) d \tau\right]
$$

where $\Delta_{n}$ is the complex superconducting order parameter of the $n$th lead without the external field. When $\Delta_{n}=0$, the $n$th lead returns to a normal state.

The Hamiltonian of the interacting central region is written in a compact form as

$$
H_{c e n}(t)=\sum_{j, \sigma} \epsilon_{j \sigma}(t) c_{j \sigma}^{\dagger} c_{j \sigma}+H_{c e n}^{i}\left(\left\{c_{j \sigma}^{\dagger}\right\},\left\{c_{j \sigma}\right\}, t, \ldots\right),
$$

where $c_{j \sigma}^{\dagger}\left(c_{j \sigma}\right)$ are the creation (annihilation) operators of the electron in the state $(j, \sigma)$ of the central region, and $H_{c e n}^{i}$ models the interactions. Note that the energy levels $\left[\epsilon_{j \sigma}(t)\right]$ of the central region can be affected by the change of the voltage on the lead, $\epsilon_{j \sigma}(t)=\epsilon_{j \sigma}^{0}+\Sigma_{n} u_{j \sigma n} \mu_{n}(t)$, with $\Sigma_{n} u_{j \sigma n}=1 .{ }^{19}$ In deriving the formal current expression, we permit that various interactions, such as the electron-electron Coulomb interaction $\sum_{j, \sigma ; j^{\prime}, \sigma^{\prime}\left(j, \sigma \neq j^{\prime}, \sigma^{\prime}\right)} U_{j \sigma, j^{\prime} \sigma^{\prime}} c_{j \sigma^{\prime}}^{\dagger} c_{j \sigma^{\prime}} c_{j^{\prime} \sigma^{\prime}}^{\dagger} c_{j^{\prime} \sigma^{\prime}}$, the electronphonon interaction $\quad \sum_{j, \sigma, q} M_{q} c_{j \sigma}^{\dagger} c_{j \sigma}\left(d_{q}^{\dagger}+d_{-q}\right)$ $+\Sigma_{q} \hbar \omega_{q} d_{q}^{\dagger} d_{q}$, etc., may exist in the central region.

The tunneling Hamiltonian $H_{T}(t)$ between leads and the central region is

$$
H_{T}(t)=\sum_{n, k, j, \sigma}\left[\mathrm{v}_{n \sigma, j}(t) a_{n k \sigma}^{\dagger} c_{j \sigma}+\mathrm{v}_{n \sigma, j}^{*}(t) c_{j \sigma}^{\dagger} a_{n k \sigma}\right]
$$

Here we allow that the external fields can also be applied to the barriers; ${ }^{20}$ then the hopping matrix element $\mathrm{v}_{n \sigma, j}(t)$ will depend on time $t$. Moreover, here we also permit some leads which have the properties: the hopping matrix element $\mathrm{v}_{n \sigma, j}(t)$ is zero, but $u_{j \sigma n}$ is not zero. These leads can model the gate terminal.

Now we perform a unitary transformation with the unitary operator $U$ as ${ }^{18}$

$$
U(t)=\exp \left[\sum_{n, k, \sigma} \frac{i}{\hbar}\left(\frac{\Phi_{n}^{0}}{2}+\int_{0}^{t} \mu_{n}(\tau) d \tau\right) a_{n k \sigma}^{\dagger} a_{n k \sigma}\right]
$$

then the Hamiltonian of the leads and the tunneling part change into 


$$
\begin{aligned}
& H_{\text {leads }}=\sum_{n, k, \sigma} \epsilon_{n k}^{0} a_{n k \sigma}^{\dagger} a_{n k \sigma} \\
& +\sum_{n, k}\left[\left|\Delta_{n}\right| a_{n k \downarrow} a_{n,-k \uparrow}+\left|\Delta_{n}\right| a_{n,-k \uparrow}^{\dagger} a_{n k \downarrow}^{\dagger}\right], \\
& H_{T}(t)=\sum_{n, k, j, \sigma}\left\{\mathrm{v}_{n \sigma, j}(t) e^{\frac{i}{\hbar}\left[\Phi_{n}^{0} / 2+\int_{0}^{t} \mu_{n}(\tau) d \tau\right]} a_{n k \sigma}^{\dagger} c_{j \sigma}+\text { H.c. }\right\},
\end{aligned}
$$

where $\Phi_{n}^{0}$ and $\left|\Delta_{n}\right|$ are the phase and the modulus of the complex superconducting order parameter of the $n$th superconducting lead. (Hereafter $\left|\Delta_{n}\right|$ will be written as $\Delta_{n}$ for simplicity.) The Hamiltonian of the central region, $H_{c e n}(t)$, is unchanged under this unitary transformation. After this transformation, both the superconducting phases and the time-dependent factors from the finite voltages appear only as a factor in the new hopping matrix elements, which makes the calculation much easier.

\section{TIME-DEPENDENT CURRENT FROM A SUPERCONDUCTING LEAD TO THE CENTRAL REGION}

\section{A. General expression for the current}

The current from the $n$th superconducting lead to the central region can be calculated from the evolution of the total number operator of the electrons in the $n$th lead, $N_{n}$ $=\Sigma_{k, \sigma} a_{n k \sigma}^{\dagger} a_{n k \sigma}$. Then one finds (in units of $\hbar=1$ )

$$
\begin{aligned}
I_{n}(t) & =-q\left\langle\dot{N}_{n}\right\rangle=i q\left\langle\left[N_{n}(t), H(t)\right]\right\rangle \\
& =2 q \operatorname{Re} \sum_{k, \sigma, j} \mathrm{v}_{n \sigma, j}(t) e^{i\left[\Phi_{n}^{0} / 2+\int_{0}^{t} \mu_{n}(\tau) d \tau\right]} i\left\langle a_{n k \sigma}^{\dagger}(t) c_{j \sigma}(t)\right\rangle \\
& \equiv I_{n \uparrow}(t)+I_{n \downarrow}(t) .
\end{aligned}
$$

In the following we only need to derive the expression of the time-dependent current with a special spin, say $I_{n \uparrow}(t)$; then the current $I_{n \downarrow}(t)$ can be easily obtained from $I_{n \uparrow}(t)$ by exchanging the up-spin and the down-spin. When the singleparticle energies $\epsilon_{j \sigma}$ and the hopping matrix elements $\mathrm{v}_{n \sigma, j}$, etc., are independent of the spin, $I_{n \downarrow}(t)$ will be equal to $I_{n \uparrow}(t)$. Because the system contains superconducting components, it is convenient to introduce the $2 \times 2$ Nambu representation in which $\mathbf{G}^{<}\left(t, t^{\prime}\right)$ and $\mathbf{G}^{r, a}\left(t, t^{\prime}\right)$ take the forms ${ }^{17}$

$$
\begin{aligned}
\mathbf{G}_{\alpha, \beta}^{r, a}\left(t, t^{\prime}\right) \equiv & \mp i \theta\left( \pm t \mp t^{\prime}\right) \\
& \times\left(\begin{array}{ll}
\left\langle\left\{X_{\alpha \uparrow}(t), Y_{\beta \uparrow}^{\dagger}\left(t^{\prime}\right)\right\}\right\rangle & \left\langle\left\{X_{\alpha \uparrow}(t), Y_{\beta \downarrow}\left(t^{\prime}\right)\right\}\right\rangle \\
\left\langle\left\{X_{\alpha \downarrow}^{\dagger}(t), Y_{\beta \uparrow}^{\dagger}\left(t^{\prime}\right)\right\}\right\rangle & \left\langle\left\{X_{\alpha \downarrow}^{\dagger}(t), Y_{\beta \downarrow}\left(t^{\prime}\right)\right\}\right\rangle
\end{array}\right),
\end{aligned}
$$

$$
\mathbf{G}_{\alpha, \beta}^{<}\left(t, t^{\prime}\right) \equiv i\left(\begin{array}{cc}
\left\langle Y_{\beta \uparrow}^{\dagger}\left(t^{\prime}\right) X_{\alpha \uparrow}(t)\right\rangle & \left\langle Y_{\beta \downarrow}\left(t^{\prime}\right) X_{\alpha \uparrow}(t)\right\rangle \\
\left\langle Y_{\beta \uparrow}^{\dagger}\left(t^{\prime}\right) X_{\alpha \downarrow}^{\dagger}(t)\right\rangle & \left\langle Y_{\beta \downarrow}\left(t^{\prime}\right) X_{\alpha \downarrow}^{\dagger}(t)\right\rangle
\end{array}\right) .
$$

Here $X$ and $Y$ represent arbitrary single-particle annihilation operators, such as $a_{n k \sigma}, c_{j \sigma}$, etc. $\alpha$ and $\beta$ are level indices as $n k, j$. In the Nambu representation, the current $I_{n \uparrow}(t)$ is given by

$$
I_{n \uparrow}(t)=2 q \operatorname{Re} \sum_{k, j} \mathrm{~V}_{n \uparrow, j}(t) e^{i\left[\Phi_{n}^{0} / 2+\int_{0}^{t} \mu_{n}(\tau) d \tau\right]} G_{j, n k ; 11}^{<}(t, t) .
$$

We next need to solve the Green function $G_{j, n k ; 11}^{<}(t, t)$. By using the Dyson equation ${ }^{2,3}$

$$
\mathbf{G}^{<}=\mathbf{g}^{<}+\mathbf{G}^{r} \boldsymbol{\Sigma}^{r} \mathbf{g}^{<}+\mathbf{G}^{r} \boldsymbol{\Sigma}^{<} \mathbf{g}^{a}+\mathbf{G}^{<} \boldsymbol{\Sigma}^{a} \mathbf{g}^{a},
$$

where $\mathbf{g}^{<, a}$ are the exact Green functions without the coupling between the leads and the central region. We assume the normal density of states $\rho_{n}^{N}(\epsilon)$ is independent of $\epsilon$ in superconducting leads, $\mathbf{g}^{<, a}$ can be expressed as ${ }^{18}$

$$
\begin{aligned}
& \sum_{k} \mathbf{g}_{n k, n k}^{<}\left(t, t^{\prime}\right) \\
& \quad=i \int d \epsilon \rho_{n}^{N}(\boldsymbol{\epsilon}) f(\boldsymbol{\epsilon}) \tilde{\rho}_{n}(\epsilon) e^{-i \epsilon\left(t-t^{\prime}\right)}\left(\begin{array}{ll}
1 & \Delta_{n} /|\epsilon| \\
\Delta_{n} /|\epsilon| & 1
\end{array}\right),
\end{aligned}
$$

$$
\sum_{k} \mathbf{g}_{n k, n k}^{a}\left(t, t^{\prime}\right)=i \theta\left(t^{\prime}-t\right) \int d \epsilon \rho_{n}^{N}(\epsilon) \frac{e^{-i \epsilon\left(t-t^{\prime}\right)}}{E_{n}^{*}}\left(\begin{array}{cc}
|\epsilon| & \Delta_{n} \\
\Delta_{n} & |\epsilon|
\end{array}\right),
$$

and $\mathbf{g}_{j, n k}^{<, r, a}\left(t, t^{\prime}\right)=0$, where $E_{n} \equiv \sqrt{\epsilon^{2}-\Delta_{n}^{2}}$, and $\tilde{\rho}_{n}(\epsilon)$ $=\theta\left(|\epsilon|-\Delta_{n}\right)|\epsilon| / E_{n}$ is the corresponding dimensionless BCS density of states; i.e., $\tilde{\rho}_{n}(\epsilon)$ is the ratio of the superconducting density of states, $\rho_{n}^{S}(\epsilon)$, to the normal density of states, $\rho_{n}^{N}(\epsilon) . f(\epsilon)$ in Eq. (13) is the Fermi distribution function, $f(\epsilon)=\left[\exp \left(\epsilon / k_{B} \mathcal{T}\right)+1\right]^{-1}$, in which $\mathcal{T}$ is the temperature. $\Sigma^{r, a,<}$ is the self-energy function,

$$
\boldsymbol{\Sigma}_{j, n k}^{r, a}\left(t, t^{\prime}\right)=\delta\left(t-t^{\prime}\right)\left(\begin{array}{lll}
\mathrm{V}_{n \uparrow, j}^{*}(t) e^{-i}\left[\Phi_{n}^{0} / 2+\int_{0}^{t} \mu_{n}(\tau) d \tau\right] & \\
0 & & -\mathrm{v}_{n \downarrow, j}(t) e^{i}\left[\Phi_{n}^{0} / 2+\int_{0}^{t} \mu_{n}(\tau) d \tau\right]
\end{array}\right),
$$


$\boldsymbol{\Sigma}_{n k, m k^{\prime}}^{r, a}\left(t, t^{\prime}\right)=0$ (for any $n k, m k^{\prime}$ ), and $\boldsymbol{\Sigma}_{\alpha, \beta}^{<}\left(t, t^{\prime}\right)=0$ (for any $\alpha, \beta)$. With the help of the Dyson equation (12), the exact Green functions $\mathbf{g}^{<, a}$, and the self-energy $\boldsymbol{\Sigma}^{r, a,<}$, we have

$$
\begin{aligned}
G_{j, n k ; 11}^{<} & \left(t, t^{\prime}\right) \\
= & \sum_{j^{\prime}} \int d t_{1}\left\{G_{j j^{\prime} ; 11}^{r}\left(t, t_{1}\right) \sum_{j^{\prime}, n k ; 11}^{r}\left(t_{1}, t_{1}\right) g_{n k, n k ; 11}^{<}\left(t_{1}, t^{\prime}\right)\right. \\
& +G_{j j^{\prime} ; 12}^{r}\left(t, t_{1}\right) \sum_{j^{\prime}, n k ; 22}^{r}\left(t_{1}, t_{1}\right) g_{n k, n k ; 21}^{<}\left(t_{1}, t^{\prime}\right) \\
& +G_{j j^{\prime} ; 11}^{<}\left(t, t_{1}\right) \sum_{j^{\prime}, n k ; 11}^{a}\left(t_{1}, t_{1}\right) g_{n k, n k ; 11}^{a}\left(t_{1}, t^{\prime}\right) \\
& \left.+G_{j j^{\prime} ; 12}^{<}\left(t, t_{1}\right) \sum_{j^{\prime}, n k ; 22}^{a}\left(t_{1}, t_{1}\right) g_{n k, n k ; 21}^{a}\left(t_{1}, t^{\prime}\right)\right\} .
\end{aligned}
$$

Substituting the expression of $G_{j, n k ; 11}^{<}(t, t)$ into Eq. (11), we note that $\mu_{n}(t)=q V_{n}+W_{n}(t)$. It is useful to define the following generalized linewidth functions:

$$
\begin{gathered}
\Gamma_{j^{\prime} j ; 11}^{n}\left(\epsilon, t_{1}, t\right)=2 \pi \rho_{n}^{N}(\epsilon) \mathrm{v}_{n \uparrow, j}(t) \mathrm{v}_{n \uparrow, j^{\prime}}^{*}\left(t_{1}\right) e^{i \int_{t_{1}}^{t} W_{n}(\tau) d \tau}, \\
\Gamma_{j^{\prime} j ; 21}^{n}\left(\epsilon, t_{1}, t\right)=2 \pi \rho_{n}^{N}(\epsilon) \mathrm{v}_{n \uparrow, j}(t) \mathrm{v}_{n \downarrow, j^{\prime}}\left(t_{1}\right) e^{i\left(\int_{0}^{t}+\int_{0}^{t_{1}}\right) W_{n}(\tau) d \tau} .
\end{gathered}
$$

In terms of these generalized linewidth functions $\Gamma^{n}\left(\epsilon, t_{1}, t\right)$, the time-dependent current $I_{n \uparrow}(t)$ can be expressed as

$$
\begin{aligned}
I_{n \uparrow}(t)= & -2 q \operatorname{Im} \sum_{j, j^{\prime}} \int_{-\infty}^{t} d t_{1} \int \frac{d \epsilon}{2 \pi} e^{-i \epsilon\left(t_{1}-t\right)} \\
& \times\left\{e ^ { i q V _ { n } ( t - t _ { 1 } ) } \Gamma _ { j ^ { \prime } j ; 1 1 } ^ { n } ( \epsilon , t _ { 1 } , t ) \left[f(\epsilon) \tilde{\rho}_{n}(\epsilon) G_{j j^{\prime} ; 11}^{r}\left(t, t_{1}\right)\right.\right. \\
& \left.+\frac{|\epsilon|}{E_{n}^{*}} G_{j j^{\prime} ; 11}^{<}\left(t, t_{1}\right)\right] \\
& -\frac{\Delta_{n}}{|\epsilon|} e^{i q V_{n}\left(t+t_{1}\right)} e^{i \Phi_{n}^{0} \Gamma_{j^{\prime} j ; 21}^{n}\left(\epsilon, t_{1}, t\right)} \\
& \left.\times\left[f(\epsilon) \tilde{\rho}_{n}(\epsilon) G_{j j^{\prime} ; 12}^{r}\left(t, t_{1}\right)+\frac{|\epsilon|}{E_{n}^{*}} G_{j j^{\prime} ; 12}^{<}\left(t, t_{1}\right)\right]\right\} .
\end{aligned}
$$

The time-dependent current expression [Eq. (19)] is the central result of this work. It describes the current in terms of local quantities: Green functions of the central region. This current formula can be used for nonlinear regions (i.e., arbitrarily high bias $V_{n}$ and arbitrarily strong external fields), multiple terminals, arbitrary interactions in the central region (e.g., the electron-electron interaction, the electron-phonon interaction, etc.), either superconducting or normal leads, and with the external fields being applied to any parts of the system. Notice that in the current formula [Eq. (19)], the anomalous Green functions $G_{12}^{r}$ and $G_{12}^{<}$emerge, which reflect the character of the superconducting leads, and lead to the Josephson current and Andreev reflection current.

If the system does not contain any superconducting components, i.e., the energy gap $\Delta_{n}$ (for all $n$ ) is zero, then the terms with anomalous Green functions vanish, and the current expression [Eq. (19)] reduces to

$$
\begin{aligned}
I_{n \uparrow}(t)= & -2 q \operatorname{Im} \sum_{j, j^{\prime}} \int_{-\infty}^{t} d t_{1} \int \frac{d \epsilon}{2 \pi} e^{-i \epsilon\left(t_{1}-t\right)} \\
& \times \Gamma_{j^{\prime} j ; 11}^{n}\left(\epsilon-q V_{n}, t_{1}, t\right)\left[f_{n}(\epsilon) G_{j j^{\prime} ; 11}^{r}\left(t, t_{1}\right)\right. \\
& \left.+G_{j j^{\prime} ; 11}^{<}\left(t, t_{1}\right)\right],
\end{aligned}
$$

where $f_{n}(\epsilon)$ is the Fermi distribution function of electrons in the $n$th lead: $f_{n}(\epsilon)=f\left(\epsilon-q V_{n}\right)=\left[\exp \left(\epsilon-q V_{n}\right) / k_{B} \mathcal{T}+1\right]^{-1}$, in which we have taken a transformation of the integral variable. When the system is only a two-terminal system and the spin of the electron is neglected, the time-dependent current [Eq. (20)], reduces to the same formula previously obtained by Wingreen and co-workers. ${ }^{2,3}$

\section{B. Case of zero-bias voltage and no external fields}

In this section, we consider the system in equilibrium, i.e., all dc voltages $V_{n}$ have the same values (set to be zero hereafter), and no external time-dependent fields applied. Then $\Gamma_{j j^{\prime} ; 11}^{n}\left(\epsilon, t_{1}, t\right)$ and $\Gamma_{j j^{\prime} ; 21}^{n}\left(\epsilon, t_{1}, t\right)$ will be independent, with time variables $t_{1}$ and $t$, and the Green functions $G_{11}^{r,<}\left(t, t_{1}\right)$ and $G_{21}^{r,<}\left(t, t_{1}\right)$ only depend on the time difference. By taking the Fourier transformation, the current formula [Eq. (19) becomes

$$
\begin{aligned}
I_{n \uparrow}= & -2 q \operatorname{Im} \sum_{j, j^{\prime}} \int \frac{d \epsilon}{2 \pi}\left\{\Gamma _ { j ^ { \prime } j ; 1 1 } ^ { n } ( \epsilon ) \left[f(\epsilon) \tilde{\rho}_{n}(\epsilon) G_{j j^{\prime} ; 11}^{r}(\epsilon)\right.\right. \\
& \left.+\frac{|\epsilon|}{2 E_{n}^{*}} G_{j j^{\prime} ; 11}^{<}(\epsilon)\right] \\
& -\frac{\Delta_{n}}{|\epsilon|} \Gamma_{j^{\prime} j ; 21}^{n}(\epsilon) e^{i \Phi_{n}^{0}}\left[f(\epsilon) \tilde{\rho}_{n}(\epsilon) G_{j j^{\prime} ; 12}^{r}(\epsilon)\right. \\
& \left.\left.+\frac{|\epsilon|}{2 E_{n}^{*}} G_{j j^{\prime} ; 12}^{<}(\epsilon)\right]\right\},
\end{aligned}
$$

where $\quad \Gamma_{j^{\prime} j ; 11}^{n}(\epsilon) \equiv 2 \pi \rho_{n}^{N}(\epsilon) \mathrm{\vee}_{n \uparrow, j} \mathrm{\vee}_{n \uparrow, j^{\prime}}^{*} \quad$ and $\quad \Gamma_{j^{\prime} j ; 21}^{n}(\epsilon)$ $\equiv 2 \pi \rho_{n}^{N}(\epsilon) \mathrm{V}_{n \uparrow, j} \mathrm{\vee}_{n \downarrow, j^{\prime}}$. In the zero-voltage case the Green function $\mathbf{G}^{<}(\boldsymbol{\epsilon})$ can be easily calculated by the following relation $^{21}$ :

$$
\mathbf{G}^{<}(\boldsymbol{\epsilon})=f(\boldsymbol{\epsilon})\left[\mathbf{G}^{a}(\boldsymbol{\epsilon})-\mathbf{G}^{r}(\boldsymbol{\epsilon})\right] .
$$

Substituting Eq. (22) into Eq. (21), and noting that $\Gamma_{j^{\prime} j ; 11}^{n}(\epsilon)=\Gamma_{j j^{\prime} ; 11}^{n *}(\epsilon)$, then the first square bracket in Eq. (21) vanishes, and we have

$$
\begin{aligned}
I_{n \uparrow}= & +2 q \operatorname{Im} \sum_{j, j^{\prime}} \int \frac{d \epsilon}{2 \pi} \frac{\Delta_{n}}{|\epsilon|} f(\epsilon)\left[\tilde{\rho}_{n}(\epsilon) G_{j j^{\prime} ; 12}^{r}(\epsilon)\right. \\
& \left.+\frac{|\epsilon|}{2 E_{n}^{*}}\left(G_{j j^{\prime} ; 12}^{a}(\epsilon)-G_{j j^{\prime} ; 12}^{r}(\epsilon)\right)\right] \Gamma_{j^{\prime} j ; 21}^{n}(\epsilon) e^{i \Phi_{n}^{0}} .
\end{aligned}
$$


In the above current expression only anomalous Green functions emerge, which means the normal single-particle tunneling does not contribute to the current, and only the Andreev reflection contributes to the dc Josephson current.

\section{DC JOSEPHSON CURRENT THROUGH A NONINTERACTING SINGLE-LEVEL CENTRAL REGION}

\section{A. Multiterminal current formula}

For a noninteracting single-level central region, its Hamiltonian $H_{c e n}$ becomes

$$
H_{c e n}=\sum_{\sigma} \epsilon_{0} c_{0 \sigma}^{\dagger} c_{0 \sigma} .
$$

Here we assume that the energy level $\epsilon_{0}$ is independent of the spin $\sigma$, and we only consider the case of zero-bias voltage and without external fields. The Green functions of the central region, $G_{00 ; 12}^{r}(\epsilon)$ and $G_{00 ; 21}^{r}(\epsilon)$ can be obtained from the Dyson equation (hereafter the subscript 00 will be omitted),

$$
\mathbf{G}^{r}(\boldsymbol{\epsilon})=\mathbf{g}^{r}(\boldsymbol{\epsilon})+\mathbf{g}^{r}(\boldsymbol{\epsilon}) \widetilde{\mathbf{\Sigma}}^{r}(\boldsymbol{\epsilon}) \mathbf{G}^{r}(\epsilon),
$$

where $\mathbf{g}^{r}(\boldsymbol{\epsilon})$ is the exact Green function of the central region without the coupling between the central region and the leads:

$$
\mathbf{g}^{r}(\boldsymbol{\epsilon})=\left(\begin{array}{ll}
\frac{1}{\epsilon-\epsilon_{0}+i 0^{+}} & 0 \\
0 & \frac{1}{\epsilon+\epsilon_{0}+i 0^{+}}
\end{array}\right),
$$

and $\widetilde{\mathbf{\Sigma}}^{r}(\boldsymbol{\epsilon})$ is the self-energy function. In the following, we make further simplifications: (1) We assume a widebandwidth approximation, i.e., we assume that the linewidths $\Gamma_{11}^{n}$ and $\Gamma_{21}^{n}$ are independent of energy. ${ }^{5}$ (2) We take the hopping matrix element $\mathrm{V}_{n \sigma, 0}$ to be real. (3) We assume $\mathrm{V}_{n \sigma, 0}$ is independent of the spin $\sigma$, leading to $\Gamma_{11}^{n}=\Gamma_{21}^{n} \equiv \Gamma^{n}$ and $I_{n \uparrow}=I_{n \downarrow}$. (4) All leads have the same energy gap $\Delta_{n}$, and will be written as $\Delta$ hereafter. Under the above simplifications, the self-energy $\widetilde{\boldsymbol{\Sigma}}(\epsilon)$ reduces to ${ }^{18}$

$$
\begin{aligned}
\widetilde{\mathbf{\Sigma}}^{r}(\boldsymbol{\epsilon})= & \sum_{n, k} \boldsymbol{\Sigma}_{0, n k}^{r} \mathbf{g}_{n k, n k}^{r} \boldsymbol{\Sigma}_{n k, 0}^{r}= \\
& -\frac{i}{2} \sum_{n} \frac{\Gamma^{n}}{\sqrt{\epsilon^{2}-\Delta^{2}}}\left(\begin{array}{ll}
|\epsilon| & -\Delta e^{-i \Phi_{n}^{0}} \\
-\Delta e^{i \Phi_{n}^{0}} & |\epsilon|
\end{array}\right) .
\end{aligned}
$$

Substituting $\mathbf{g}^{r}(\boldsymbol{\epsilon})$ and $\widetilde{\mathbf{\Sigma}}^{r}(\boldsymbol{\epsilon})$ into the Dyson equation [Eq. (25)], one easily obtains the Green function $\mathbf{G}^{r}(\boldsymbol{\epsilon})$ as

$$
\mathbf{G}^{r}(\boldsymbol{\epsilon})=\frac{1}{B}\left(\begin{array}{ll}
\left(g_{22}^{r-1}-\widetilde{\Sigma}_{22}^{r}\right) & \widetilde{\Sigma}_{12}^{r} \\
\widetilde{\Sigma}_{21}^{r} & \left(g_{11}^{r-1}-\widetilde{\Sigma}_{11}^{r}\right)
\end{array}\right),
$$

where the notation $B(\epsilon)$ is defined by

$$
B(\epsilon)=\left(g_{11}^{r-1}-\widetilde{\Sigma}_{11}^{r}\right)\left(g_{22}^{r-1}-\widetilde{\Sigma}_{22}^{r}\right)-\widetilde{\Sigma}_{12}^{r} \widetilde{\Sigma}_{21}^{r} .
$$

Then substituting the Green functions $G_{12}^{r}(\epsilon)$ and $G_{21}^{r *}(\epsilon)$ into the current $I_{n \uparrow}$ [Eq. (23)], one can derive the current $I_{n}$ $\left(I_{n}=I_{n \uparrow}+I_{n \downarrow}=2 I_{n \uparrow}\right)$ as

$$
I_{n}=2 q \int \frac{d \epsilon}{2 \pi} \frac{\Gamma^{n} \Delta^{2}}{\epsilon^{2}-\Delta^{2}} f(\epsilon) \operatorname{Im} \frac{1}{B^{*}(\epsilon)} \sum_{m} \Gamma^{m} \sin \left(\Phi_{n}^{0}-\Phi_{m}^{0}\right) .
$$

This current expression can be used to calculate the dc Josephson current through a noninteracting single-level central region coupled to superconducting leads. The current $I_{n}$ can be split into two parts: ${ }^{16,22} I_{n}=I_{n}^{c}+I_{n}^{d}$, where $I_{n}^{c}$ is from the continuous spectrum for $|\epsilon|>\Delta$, in which $B$ has a imaginary part; and $I_{n}^{d}$ originates from the discrete spectrum for $|\epsilon|<\Delta$, in which the imaginary part of $B$ is a positive infinitesimal $\left(i 0^{+}\right)$.

\section{B. Two-terminal system}

In the following numerical calculation, we take symmetric barriers, i.e., $\Gamma^{1}=\Gamma^{2} \equiv \Gamma$; and zero temperature $(\mathcal{T}=0)$. Then the current $I_{1}^{c}$ reduces to

$$
I_{1}^{c}=2 q\left(\int_{-\infty}^{-\Delta}+\int_{\Delta}^{\infty}\right) \frac{d \epsilon}{2 \pi} \frac{f(\epsilon)(\Gamma)^{3} \Delta^{2}}{|B|^{2}\left(\epsilon^{2}-\Delta^{2}\right)} 2 \epsilon \tilde{\rho}(\epsilon) \sin (\delta \Phi),
$$

where $\delta \Phi=\Phi_{1}^{0}-\Phi_{2}^{0}$. For $|\epsilon|>\Delta, B$ becomes

$$
B=\epsilon^{2}-\epsilon_{0}^{2}+2 i \epsilon \tilde{\rho}(\epsilon) \Gamma-(\Gamma)^{2}-\frac{(\Gamma)^{2} \Delta^{2}}{2\left(\epsilon^{2}-\Delta^{2}\right)}[1-\cos (\delta \Phi)] .
$$

The current $I_{1}^{d}$ reduces to

$$
I_{1}^{d}=\frac{q(\Gamma)^{2} \Delta^{2}}{\Delta^{2}-\widetilde{\epsilon}_{0}^{2}} \frac{\sin (\delta \Phi)}{2 \sqrt{\epsilon_{0}^{2}+\frac{(\Gamma)^{2} \Delta^{2}[1+\cos (\delta \Phi)]}{2\left(\Delta^{2}-\widetilde{\epsilon}_{0}^{2}\right)}}},
$$

where $\tilde{\epsilon}_{0}$ is the renormalized energy level, which can be obtained from the equation

$$
\tilde{\epsilon}_{0}=\frac{-\Gamma\left|\tilde{\epsilon}_{0}\right|}{\sqrt{\Delta^{2}-\tilde{\epsilon}_{0}^{2}}}-\sqrt{\epsilon_{0}^{2}+\frac{(\Gamma)^{2} \Delta^{2}[1+\cos (\delta \Phi)]}{2\left(\Delta^{2}-\tilde{\epsilon}_{0}^{2}\right)}},
$$

with $-\Delta<\tilde{\epsilon}_{0}<0$.

In this case the dc Josephson current (includes both $I^{c}$ and $I^{d}$ ) originates from Andreev reflection at the interface between the superconducting leads and the central region. Figure 2 shows the current $I_{1}^{c}$ (from the continuous spectrum) vs the central region's energy level $\epsilon_{0}$, which can be controlled by the gate voltage. Two peaks emerge around $\epsilon_{0}= \pm \Delta$, due to the fact that the Andreev reflection probability is one at $\epsilon= \pm \Delta .{ }^{23,24}$ Figure 3 shows the current $I_{1}^{d}$ (from the discrete spectrum) vs $\epsilon_{0}$ for the same parameters as in Fig. 2. One peak emerges at $\epsilon_{0}=0$, because the weight of the renormalized discrete level $\tilde{\epsilon}_{0}$ is proportional to 


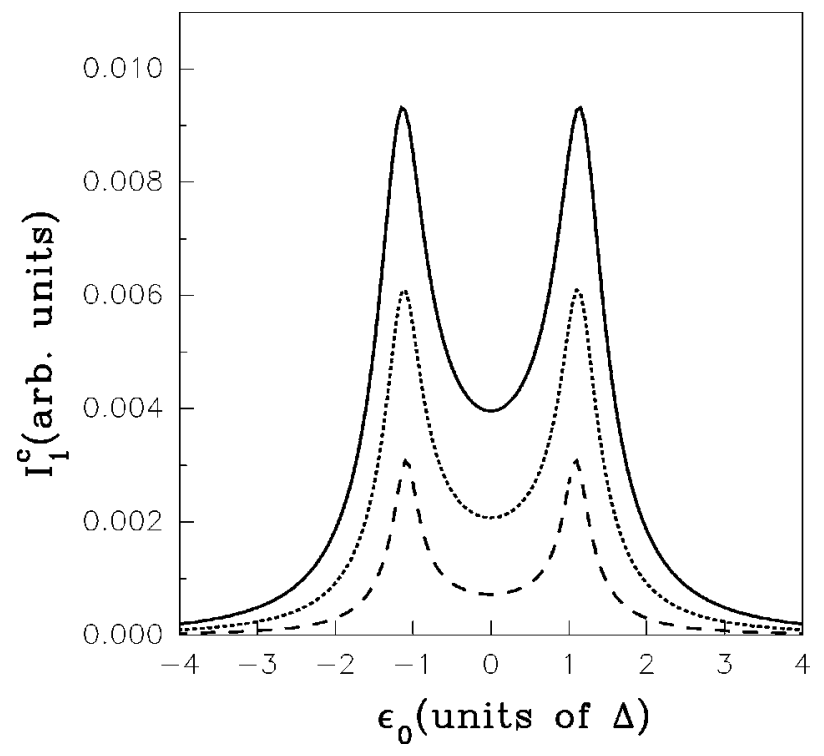

FIG. 2. dc Josephson current $I_{1}^{c}$ (from the continuous spectrum) vs the energy level of the central region, $\epsilon_{0}$, with the phase difference $\delta \Phi=3 \pi / 2$ and the energy gap $\Delta=1$. The solid, dotted, and dashed curves correspond to $\Gamma=0.1,0.07$, and 0.04 , respectively.

$$
\left(\epsilon_{0}^{2}+\frac{(\Gamma)^{2} \Delta^{2}[1+\cos (\delta \Phi)]}{2\left(\Delta^{2}-\tilde{\epsilon}_{0}^{2}\right)}\right)^{-1 / 2}
$$

which has the largest value while $\epsilon_{0}=0$. When $\tilde{\epsilon}_{0}$ approaches the gap $\Delta$, the current $I_{1}^{d}$ rapidly increases. Then with $\left|\epsilon_{0}\right|$ increasing further, Eq. (34) does not have a solution between $-\Delta$ and 0 ; i.e., there is no discrete spectrum, leading to $I_{1}^{d}=0$. Notice that the scales are different in Figs. 2 and 3 ; in fact the current $I_{1}^{d}$ is about eight times larger than the current $I_{1}^{c}$.

Now we investigate dc Josephson current $I_{1}$ vs the phase difference $\delta \Phi$. For $\Gamma<\Delta$ (the weak-coupling case), the curve of the current $I_{1}^{c}$ vs $\delta \Phi$ is similar to a sine curve.

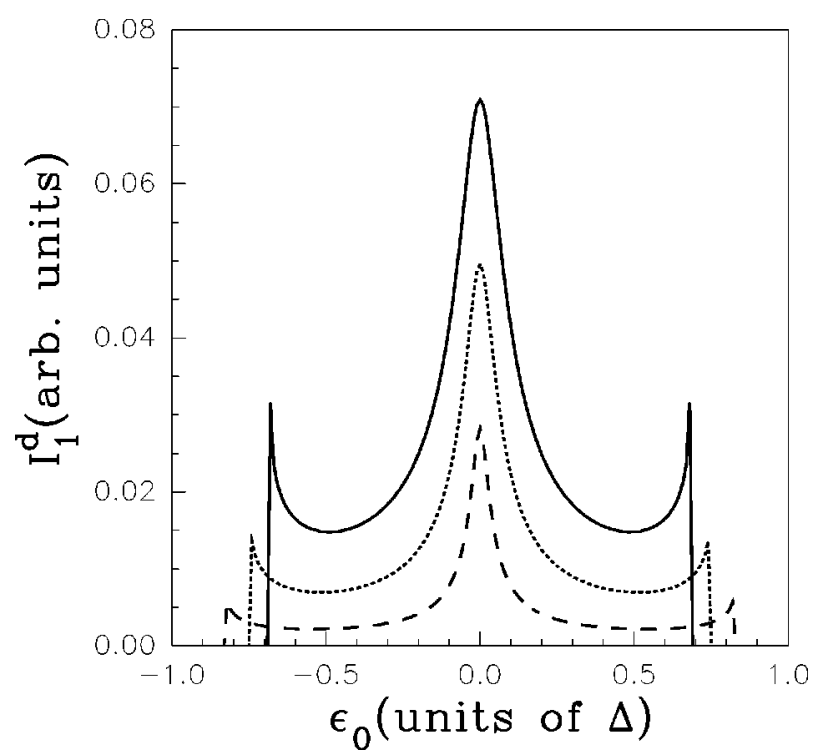

FIG. 3. dc Josephson current $I_{1}^{d}$ (from the discreted spectrum) vs the energy level $\epsilon_{0}$, with $\delta \Phi=\pi / 2$. The other parameters are the same as in Fig. 2.

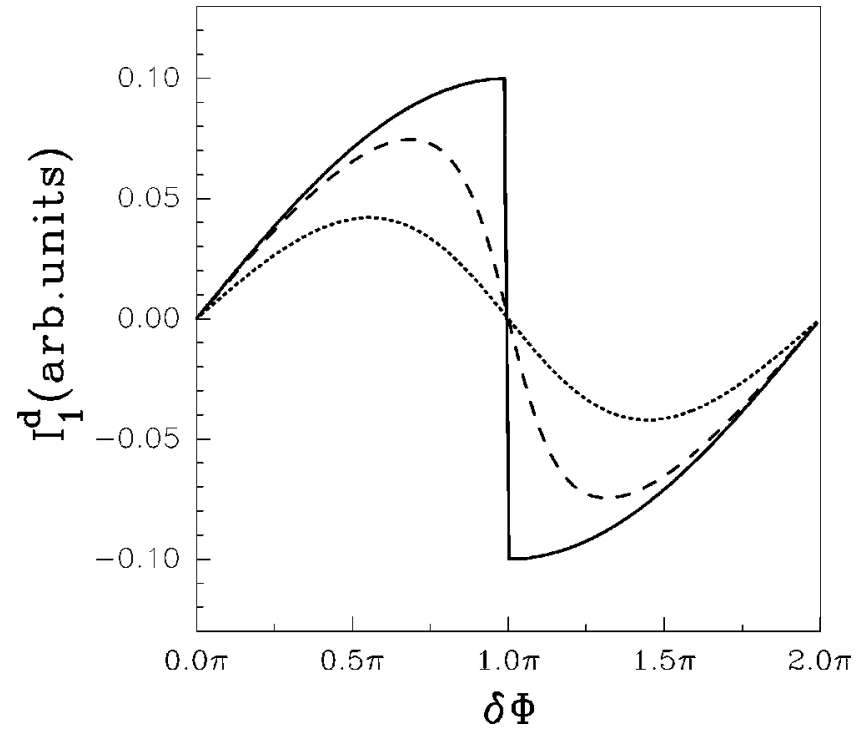

FIG. 4. dc Josephson current $I_{1}^{d}$ vs the phase difference $\delta \Phi$ for different values of the energy level $\epsilon_{0}$, with $\Gamma=0.1$ and $\Delta=1$. The solid, dashed, and dotted curves correspond to $\epsilon_{0}=0,0.03$, and 0.1 , respectively.

However, for $\Gamma \sim \Delta$, or $\Gamma>\Delta$, the shape of the curve slightly deviates from the sine curve (not shown here). The current $I_{1}^{d}$ vs $\delta \Phi$ for different values of the energy level $\epsilon_{0}$ is shown in Fig. 4. When $\epsilon_{0}=0$, the current $I_{1}^{d}$ abruptly changes at $\delta \Phi$ $=\pi$, which is similar to the $S-N-S$ system without the barriers at the $N-S$ interfaces. ${ }^{16}$ With $\epsilon_{0}$ apart from zero, the abrupt jump will be suppressed, and gradually approach a sinelike curve.

It should be mentioned that the current $I_{1}^{c}$ is a $\pi$-junction Josephson relation, ${ }^{25,26}$ i.e., $I_{1}^{c}=I_{1 c}^{c} \sin (\delta \Phi+\pi)$, not $I_{1}^{c}$ $=I_{1 c}^{c} \sin \delta \Phi$, and $I_{1}^{c}$ is negative at $\delta \Phi=\pi / 2$. However, the current $I_{1}^{d}$ is a general Josephson relation.

\section{Three-terminal system}

Now we investigate the dc Josephson current $I_{n}$ in the three-terminal system. From Eqs. (29) and (30), if all phases $\Phi_{n}^{0}$ of superconducting leads change by the same amount, the current $I_{n}$ does not change. Therefore we can set one of the phases of leads equal to zero, say $\Phi_{1}^{0}=0$. Figure 5 shows the current $I_{1}^{c}$ vs the phase $\Phi_{2}^{0}$ for $\Gamma<\Delta$ with different values of $\Phi_{3}^{0}$. All curves are similar to the sine curve, but shifted with each other a certain approximate value. In fact, the current $I_{1}^{c}$ can be expressed approximately as

$$
I_{1}^{c}=I_{21}^{c} \sin \left(\Phi_{1}^{0}-\Phi_{2}^{0}\right)+I_{31}^{c} \sin \left(\Phi_{1}^{0}-\Phi_{3}^{0}\right) .
$$

For $\Gamma>\Delta$, the current $I_{1}^{c}$ cannot be written as two separated parts, as mentioned above. In particular, curves of $I_{1}^{c}$ vs $\Phi_{2}^{0}$ will be different from the sine curve for some values of $\Phi_{3}^{0}$ (see Fig. 6).

\section{CONCLUSIONS}

In this paper, we have obtained an extended general timedependent current formula through an interacting central region coupled to two normal leads obtained by Wingreen and 


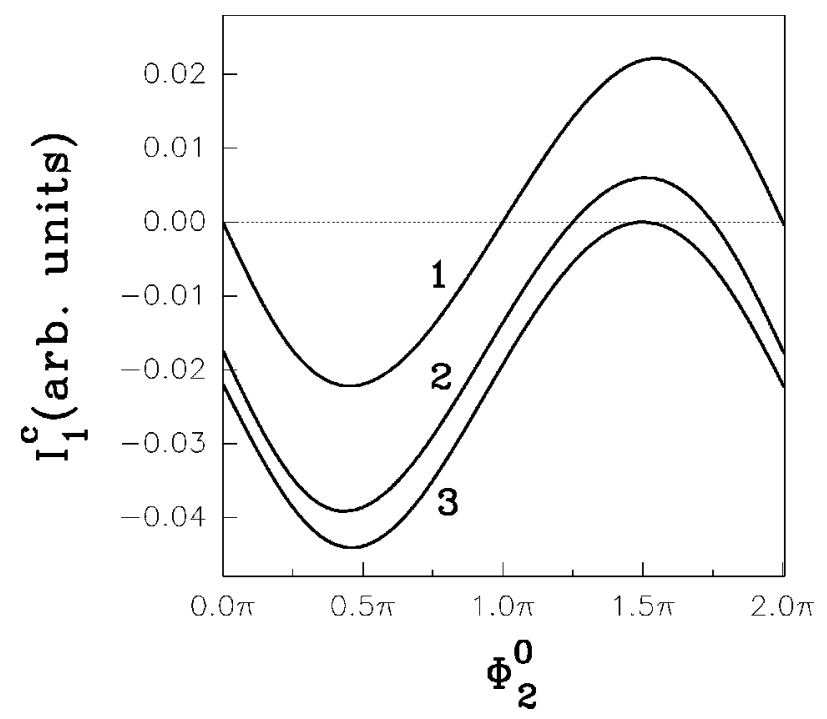

FIG. 5. dc Josephson current $I_{1}^{c}$ vs the phase of the second superconducting lead $\Phi_{2}^{0}$ in a three-terminal system for $\Gamma<\Delta$. The parameters are $\Gamma=0.3, \Delta=1$, and $\epsilon_{0}=-1$, and curves 1,2 , and 3 correspond to $\Phi_{3}^{0}=0, \pi / 4$, and $\pi / 2$, respectively.

co-workers, ${ }^{2,3}$ to systems with multiterminal superconducting leads, i.e., to mesoscopic hybrid multiterminal systems. This current formula can be used to study nonlinear electron transport through an interacting central region in the presence of time-dependent external fields applied to any parts of the system. As an application of this current formula, we investigated the dc Josephson current for a noninteracting single-level central region. For a two-terminal system, we studied the properties of the dc Josephson current, and discussed in detail the behavior of its two parts: $I_{1}^{c}$ from the continuous spectrum, and $I_{1}^{d}$ from the discrete spectrum. For a three-terminal system, we investigated the current-phase relation, and, in particular, the difference between the cases $\Gamma<\Delta$ and $\Gamma>\Delta$.

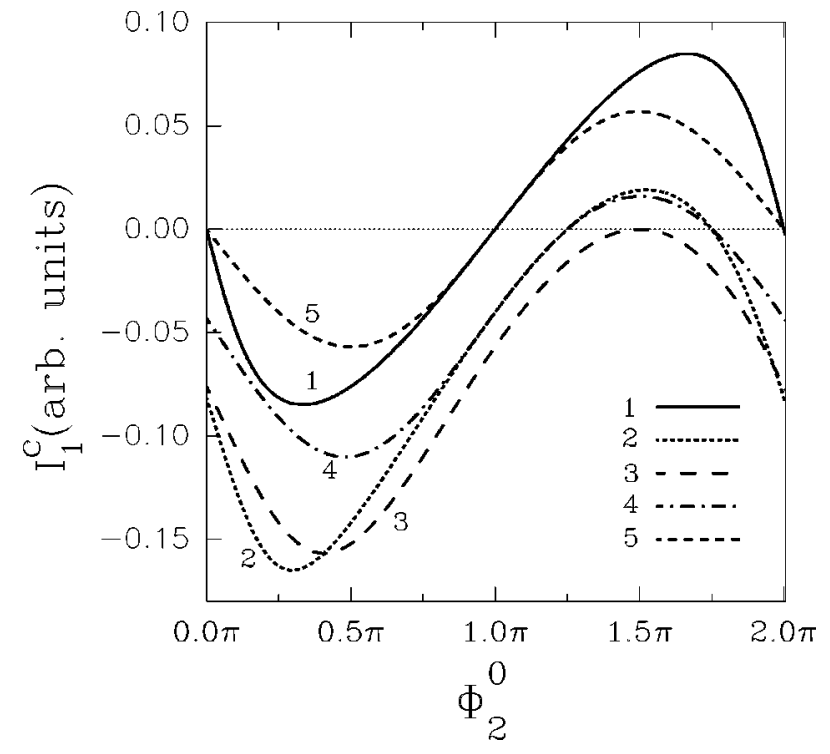

FIG. 6. dc Josephson current $I_{1}^{c}$ vs the phase $\Phi_{2}^{0}$ in a threeterminal system for $\Gamma>\Delta$. The parameters are $\Gamma=2, \Delta=1$, and $\epsilon_{0}=-1$, and curves $1-5$ correspond to $\Phi_{3}^{0}=0, \pi / 4, \pi / 2,3 \pi / 4$, and $\pi$, respectively.

\section{ACKNOWLEDGMENTS}

We gratefully acknowledge financial support from the RGC from the SAR Government of Hong Kong under Grant No. HKU 7215/99P, a grant from the Croucher Foundation, a CRCG grant from the University of Hong Kong, and a research grant from the Chinese National Natural Science Foundation, and the Doctoral Program Foundation of the Institution of Higher Education. We also thank the Computer Center of the University of Hong Kong for computational facilities. Q.F.S. and T.H.L. would like to thank Q. R. Zheng, X. A. Zhao, and Y. D. Wei for many helpful discussions.
*Author to whom correspondence should be addressed. Permanent address: Department of Physics, Peking University, Beijing 100871, China.

${ }^{1}$ G. Garcia-Calderon, in The Physics of Low-Dimensional Semiconductor Structures, edited by P. Butcher, N. H. March, and M. P. Tosi (Plenum, New York, 1993), p. 267.

${ }^{2}$ N. S. Wingreen, Antti-Pekka Jauho, and Y. Meir, Phys. Rev. B 48, 8487 (1993).

${ }^{3}$ Antti-Pekka Jauho, N. S. Wingreen, and Y. Meir, Phys. Rev. B 50, 5528 (1994).

${ }^{4}$ L. P. Kouwenhoven, S. Jauhar, J. Orenstein, P. L. McEuen, Y. Nagamune, J. Motohisa, and H. Sakaki, Phys. Rev. Lett. 73, 3443 (1994); T. H. Oosterkamp, L. P. Kouwenhoven, A. E. A. Koolen, N. C. van der Vaart, and C. J. P. M. Harmans, Phys. Rev. Lett. 78, 3169 (1997).

${ }^{5}$ Q.-F. Sun, J. Wang, and T.-H. Lin, Phys. Rev. B 58, 2008 (1998); ibid. 58, 13007 (1998).

${ }^{6}$ T. H. Oosterkamp, T. Fujisawa, W. G. van der Wiel, K. Ishibashi, R. V. Hijman, S. Tatucha, and L. P. Kouwenhoven, Nature (London) 395, 873 (1998); H. Drexler, J. S. Scott, S. J. Allen, K.
L. Campman, and A. C. Gossard, Appl. Phys. Lett. 67, 2816 (1995).

${ }^{7}$ B. J. Keay, S. J. Allen, Jr., J. Galan, J. P. Kaminski, K. L. Campman, A. C. Gossard, U. Bhattacharya, and M. J. W. Rodwell, Phys. Rev. Lett. 75, 4098 (1995); B. J. Keay, S. Zeuner, S. J. Allen, Jr., K. D. Maranowski, A. C. Gossard, U. Bhattacharya, and M. J. W. Rodwell, Phys. Rev. Lett. 75, 4102 (1995).

${ }^{8}$ C. J. Lambert and R. Raimondi, J. Phys.: Condens. Matter 10, 901 (1998).

${ }^{9}$ A. W. Kleinsasser, R. E. Miller, W. H. Mallison, and G. B. Arnold, Phys. Rev. Lett. 72, 1738 (1994); N. Van der Post, E. T. Peters, I. K. Yanson, and J. M. van Ruitenbeck, Phys. Rev. Lett. 73, 2611 (1994).

${ }^{10}$ A. F. Morpurgo, B. J. van Wees, T. M. Klapwij, and G. Borghs, Phys. Rev. Lett. 79, 4010 (1997).

${ }^{11}$ M. T. Tuominen, J. M. Hergenrother, T. S. Tighe, and M. Tinkham, Phys. Rev. Lett. 69, 1997 (1992); T. M. Eiles, J. M. Martinis, and M. H. Devoret, Phys. Rev. Lett 70, 1862 (1993); F. W. J. Hekking, L. I. Glazman, K. A. Matveev, and R. I. Shekhter, Phys. Rev. Lett. 70, 4138 (1993). 
${ }^{12}$ F. Sols and J. Ferrer, Phys. Rev. B 49, 15913 (1994).

${ }^{13}$ M. Hurd, S. Datta, and P. F. Bagwell, Phys. Rev. B 56, 11232 (1997).

${ }^{14}$ S. Ishizaka, J. Sone, and T. Ando, Phys. Rev. B 52, 8358 (1995).

${ }^{15}$ R. Bauernschmitt, J. Siewert, Yu. V. Nazarov, and A. A. Odintsov, Phys. Rev. B 49, 4076 (1994).

${ }^{16}$ P. F. Bagwell, Phys. Rev. B 46, 12573 (1992).

${ }^{17}$ J. C. Cuevas, A. Martin-Rodero, and A. Levy Yeyati, Phys. Rev. B 54, 7366 (1996).

${ }^{18}$ Q.-F. Sun, J. Wang, and T.-H. Lin, Phys. Rev. B 59, 13126 (1999).

${ }^{19}$ Here $\Sigma_{n} u_{j \sigma n}=1$ is the result of the gauge invariance. For more discussion of gauge invariance, see Z. S. Ma, J. Wang, and H. Guo, Phys. Rev. B 57, 9108 (1998); M. H. Pedersen and M.
Büttiker, ibid. 58, 12993 (1998).

${ }^{20}$ Q.-F. Sun and T.-H. Lin, J. Phys.: Condens. Matter 9, 3043 (1997).

${ }^{21}$ A. Levy Yeyati, A. Martin-Rodero, and F. J. Garcia-Vidal, Phys. Rev. B 51, 3743 (1995).

${ }^{22}$ M. Hurd and G. Wendin, Phys. Rev. B 51, 3754 (1995).

${ }^{23}$ G. E. Blonder, M. Tinkham, and T. M. Klapwijk, Phys. Rev. B 25, 4515 (1982).

${ }^{24}$ Q.-F. Sun, J. Wang, and T.-H. Lin, Phys. Rev. B 59, 3831 (1999).

${ }^{25}$ Th. Mühge, N. N. Garif'yanov, Yu. V. Goryunov, G. G. Khaliullin, L. R. Tagirov, K. Westerholt, I. A. Garifullin, and H. Zabel, Phys. Rev. Lett. 77, 1857 (1996).

${ }^{26}$ D. J. van Harlingen, Rev. Mod. Phys. 67, 515 (1995). 